\title{
Segregación escolar por nivel socioeconómico en educación secundaria en Perú y sus regiones
}

\author{
F. Javier Murillo \\ Universidad Autónoma de Madrid \\ javier.murillo@uam.es \\ Sandra Carrillo \\ Pontificia Universidad Católica del Perú \\ carrillo.sandra@pucp.pe
}

Recibido: 10/03/2019

Aprobado: 25/07/2019 


\title{
Segregación escolar por nivel socioeconómico en educación secundaria en Perú y sus regiones
}

\begin{abstract}
Resumen
Diferentes estudios internacionales han evidenciado que Perú es uno de los países de América Latina con mayor segregación escolar. El objetivo de este estudio es profundizar en esa afirmación y estimar la magnitud de la segregación de las escuelas de secundaria por nivel socioeconómico en el total de Perú y en sus regiones con una mirada específica en las escuelas situadas en contextos urbanos. Para ello, se realiza una explotación especial de los datos de la Evaluación Censal de Estudiantes del 2016 del Ministerio de Educación. Se utilizan los índices de Gorard y de aislamiento, y la variable criterio es el índice socioeconómico. La muestra estuvo conformada por 489.161 estudiantes de 25 regiones. Los resultados confirman que la magnitud de la segregación escolar en las escuelas de secundaria es alta y se encuentra una tendencia a una mayor segregación en los grupos de menor nivel socioeconómico, así como diferencias entre las regiones y entre cada grupo minoritario. El estudio muestra la necesidad de seguir profundizando en este tipo de estudios, puesto que permite contar con evidencia para atender, de manera urgente y diferenciada, a los grupos con mayor segregación escolar. De este modo, es posible garantizar la igualdad de oportunidades a todos los estudiantes del país.

Palabras clave: Segregación escolar, nivel socioeconómico, educación básica, Perú, equidad educativa
\end{abstract}

\section{Socio-economic school segregation in secondary education in Peru and its regions}

\begin{abstract}
International studies have shown that Peru is one of the Latin American countries with the highest school segregation. This study aims to estimate the magnitude of the socio-economic school segregation in Peru and its regions, specifically in secondary schools located in urban contexts. Based on the Census Evaluation of Students of 2016 by the Ministry of Education, we carried out a data mining process estimating the Gorard segregation and the isolation indices and using the socioeconomic index as a criterion variable. The sample was composed by 489.161 students from 25 regions. The results confirm that the magnitude of school segregation in secondary schools is high and that there is a trend towards greater segregation in lower socio-economic groups, as well as differences between regions and between each minority group. Also, the study shows the need to continue deepening this type of studies, since it allows to have evidence to respond, in an urgent and differentiated way, to the groups with higher school segregation. This way, it is possible to guarantee equal opportunities to all students in the country.
\end{abstract}

Keywords: School segregation, socioeconomic level, basic education, Peru,

educational equity 


\section{Introducción ${ }^{1}$}

Recientes investigaciones han aportado sólidas evidencias que defienden que el Perú es uno de los países con mayor segregación escolar de América Latina, tanto en lo que se refiere a educación primaria (Murillo, 2016; Murillo y Martínez-Garrido, 2017a) como a educación secundaria (Benavides, León y Etesse, 2014; Krüger, 2019; Murillo, Duk y Martínez-Garrido, 2018; Vázquez, 2012). A partir de ello, quizá, se pueda hablar de Perú como uno de los países con la mayor segregación escolar del mundo.

Es cierto que en, las últimas dos décadas, el Estado peruano ha incrementado la inversión en educación, y, así, ha mejorado los indicadores de cobertura (tasas de matrícula, conclusión, deserción, repitencia y atraso) en todos los niveles (inicial, primaria, secundaria y superior) y los logros de aprendizaje, principalmente, en el nivel de primaria. En el caso específico de la secundaria, si bien al 2015 las mejoras han sido importantes en matrícula $(83,4 \%)$, conclusión $(71,4 \%)$, deserción escolar $(7,8 \%)$, repitencia $(3,3 \%)$ y atraso $(11,2 \%)$, estas no han sido suficientes (OECD, 2017). Asimismo, la Evaluación Censal de Estudiantes (ECE) del 2015 para segundo de secundaria evidencia que menos del 15\% de estudiantes se encuentran en un nivel satisfactorio de aprendizaje. Dicha información se corrobora con el Programa de Evaluación Internacional de Estudiantes (PISA) aplicado en el 2000, 2009 y 2012, en el que - a pesar de las mejoras en los últimos años- el Peru se ubica entre los últimos países de América Latina (Ganimian, 2015; OECD, 2017).

Sumado a lo anterior, diferentes estudios sobre la inequidad del sistema educativo peruano y sus desigualdades han evidenciado que se profundiza la brecha en los indicadores de cobertura y calidad con estudiantes pobres o pobres extremos, indígenas o que viven en zonas rurales, y estudiantes con alguna discapacidad (Banco Mundial, 2001; Bello y Villarán, 2004; Cuenca, 2015; Cueto, 2004; Cueto, León y Muñoz, 2014; De Belaunde, 2011; Ganimian, 2015; Guadalupe, Burga, Miranda y Castillo, 2015; Guadalupe, León, Rodríguez y Vargas, 2017; Guadalupe, Castillo, Castro, Villanueva y Urquizo, 2016; Ministerio de Educación, 2006, entre otros). La segregación escolar genera y refuerza estas desigualdades.

Así, la educación no es solo importante para mejorar la competitividad y el crecimiento económico, sino que también se trata de un "mecanismo generador de mayor cohesión social, de cultura democrática y de mejores prácticas institucionales (good governance)" (Bonal, 2006, p. 13). Por ello, los sistemas educativos altamente segregados tienen consecuencias muy desfavorables para la población escolar y el conjunto de la sociedad, tanto en el desarrollo personal y social de los estudiantes, como en los aprendizajes. Estas consecuencias

1. Este artículo forma parte de una línea de investigación desarrollada por ambos autores sobre la segregación escolar en el Perú en el marco del Programa de Doctorado en Educación de la Universidad Autónoma de Madrid. 
afectan, en especial, a aquellos más vulnerables o de sectores desfavorecidos, como a aquellos que proceden de familias de mayores recursos, al no formarse en espacios más inclusivos y diversos.

Este artículo busca contribuir a la discusión al estimar la magnitud de la segregación escolar por nivel socioeconómico en el Perú, a partir de una explotación especial de las bases de datos de la Evaluación Censal de Estudiantes (ECE). Así, es posible realizar un análisis a mayor profundidad de la segregación, por su nivel censal, tanto para todas las escuelas del nivel secundaria del país, según regiones, así como para las escuelas situadas en contextos urbanos.

\section{Marco teórico}

La noción de segregación escolar hace referencia a la distribución desigual de los estudiantes en las escuelas, en función de sus características personales, culturales o sociales (Dupriez 2010; Ireson y Hallam, 2001; Murillo, 2016; Vázquez, 2012). Si bien esta noción es multidimensional, son dos las dimensiones más utilizadas en la literatura: la de uniformidad y la de exposición. La dimensión de uniformidad hace referencia a la distribución desbalanceada de los alumnos entre escuelas en función de sus características personales, culturales o sociales, especialmente en términos de su nivel socioeconómico, grupo étnico de pertenencia, cultura u origen nacional (Bellei, 2013; Jaume y Gasparini, 2013; Murillo y Martínez-Garrido, 2017a, 2018; Valenzuela, Bellei y De Los Ríos, 2010). La dimensión de exposición se refiere a la probabilidad de que los estudiantes convivan con estudiantes de diferentes grupos en las escuelas (Bellei, 2013; Dupriez, 2010; Murillo, Duk y Martínez-Garrido, 2018).

La segregación escolar afecta el aprendizaje de los estudiantes, porque las características socioeconómicas de los alumnos influyen en los aprendizajes. Así, este fenómeno produce desigualdades en cuanto al acceso de cada grupo a determinada calidad educativa. Es decir, si el desempeño de los estudiantes en la escuela depende de sus compañeros, los niveles más altos de segregación social conducirían a una mayor desigualdad en el rendimiento académico y, por lo tanto, a una mayor desigualdad en los resultados posteriores (Durlauf, 2006; Jaume y Gasparini, 2013; Jenkins, Micklewright y Schnepf, 2006). De esta forma, una mayor segregación social puede incluso reducir los niveles promedio de rendimiento, dado que la segregación de las personas vulnerables agrega a su vulnerabilidad individual la vulnerabilidad colectiva de su grupo. Este hecho afecta directamente el diseño de las políticas educativas, en tanto dificulta la posibilidad de aplicar programas exitosos de mejoramiento escolar a un nivel institucional en el largo plazo, lo cual hace a los grupos aún más vulnerables, y supone el riesgo de instalar fenómenos de exclusión y desintegración crónicos (Bonal y Bellei, 2018; Valenzuela, Bellei y De Los Ríos, 2010).

Asimismo, la segregación debilita el rol cohesionador de la escuela como espacio de integración social. La escuela es el lugar donde los estudiantes apren10I den a convivir en la diversidad económica, social y cultural. La segregación 
impide este proceso, pues contribuye a la generación de fenómenos de exclusión y desintegración en la sociedad, lo cual puede, incluso, convertirse en una amenaza para la cohesión social (Bonal y Bellei, 2018; Durlauf, 2006; Gasparini, Jaume, Serio y Vázquez, 2011). Además, si los grupos que se forman en las escuelas son una manifestación de alguna forma de segregación, estos pueden dar lugar a desigualdades persistentes y trampas de pobreza a través de comportamientos de imitación "negativos" (Cueto, León y Miranda, 2016; Durlauf, 2006; Valenzuela et al., 2010; Vázquez, 2012).

Los primeros estudios sobre segregación escolar se iniciaron en Estados Unidos, a partir del fallo de la Corte Suprema en el año 1954, que declaró inconstitucionales las leyes que establecían escuelas públicas separadas para estudiantes de raza negra y blanca. Décadas después, surgieron los estudios sobre segregación escolar de carácter socioeconómico orientados a demostrar la distribución desigual de los estudiantes en las escuelas en función del nivel socioeconómico y cultural de sus familias (Bonal y Bellei, 2018; Murillo y Martínez-Garrido, 2017b).

Aunque se reconoce la existencia de una alta segregación en América Latina desde hace décadas, el interés por investigarla es reciente y aún son escasos los estudios que abordan su medición. Como antecedentes, Reimers (2000) y Unesco $(2008,2010)$ evidenciaron la alta estratificación de las escuelas de la región. En Argentina, destacan los estudios desarrollados por Gasparini, Jaume, Serio y Vázquez (2011); Jaume y Gasparini (2013); y Krüger (2011, 2014, 2018). En Brasil, se encuentra la investigación de Lisboa Bartholo y da Costa (2018); y, en Chile, de Bellei (2007, 2013); Bellei, Contreras, Canales y Orellana (2018); Elacqua (2009, 2012); Rojas (2009); y Valenzuela, Bellei y De Los Ríos (2008, 2010, 2014). En Colombia, cabe mencionar los trabajos de Rangel (2006), y Rangel y Lleras (2010); en Ecuador, el de Murillo y Martínez-Garrido (2017b); y, en México, el estudio de Cárdenas Denham (2011). Por último, en Perú, destacan las investigaciones de Benavides, León y Etesse (2014); Balarín (2015, 2016); Balarín y Escudero (2018); Cueto, León y Miranda (2016); y el de León y Collahua (2016). Todos estos trabajos confirman que América Latina tiene sistemas educativos altamente segregados.

Especialmente interesantes son los estudios que abordan la segregación desde una perspectiva comparada. En el nivel de educación primaria, Murillo (2016), y Murillo y Martínez-Garrido (2017a) presentan estimaciones de la segregación escolar por nivel socioeconómico en América Latina usando los datos del Tercer Estudio Comparativo y Explicativo (Terce) de Unesco (2013). En el primero de ellos, Murillo presenta una serie de índices para estimar la magnitud de la segregación escolar (disimilitud, Gorard, de aislamiento, de raíz cuadrada y de inclusión socioeconómica) y evidencia una alta segregación escolar en los países de la región. El segundo trabajo confirma estos resultados utilizando el índice de disimilitud. Ambos estudios muestran que Perú es uno de los países con mayor nivel de segregación socioeconómica en la región. 
Los estudios comparados en el nivel de Educación Secundaria utilizan los datos del Programa de Evaluación Internacional de Estudiantes (PISA) aplicado en sus diferentes versiones (entre los años 2000 y 2015). En ese marco, destaca el estudio de Vázquez (2012), quien realiza un análisis de la segregación por nivel socioeconómico con los datos de PISA 2009 y su evolución entre los años 2000 y 2009, utilizando el índice de disimilitud con la mediana de estudiantes como grupo minoritario. Los resultados evidencian claramente dos extremos. En uno, se encuentran los menores índices de segregación (entre 0,26 y 0,31 ), en que se ubican los países escandinavos; y, en el otro, los países más segregados (con valores de más de 0,5): Perú, Chile y Tailandia. En este estudio, se determinó que Perú había aumentado en 0,09 puntos el valor de su índice de disimilitud en el período estimado.

Basándose en los datos de PISA 2000 y 2009, Benavides, León y Etesse (2014) utilizan el índice de Hutchens y confirman un incremento de la segregación por nivel socioeconómico en las escuelas del Perú. Dicho estudio identifica que la brecha entre los extremos socioeconómicos se amplía entre un período y otro, a pesar de que esta se reduce para los quintiles intermedios.

Murillo, Duk y Martínez Garrido (2018), por su parte, utilizan los datos de las cinco ediciones de PISA para analizar la evolución de la magnitud de la segregación escolar por nivel socioeconómico en diez países de América Latina. Por medio de los índices de Gorard y de aislamiento, los investigadores encuentran que, en la mayoría de los países, los casos en los que la segregación disminuye corresponden a aquellos de estudiantes con familias de mayor nivel socioeconómico, mientras que la segregación se incrementa en los de menor nivel. Sin embargo, el Perú es el país que presenta una de las mayores tasas de segregación escolar por nivel socioeconómico con una tendencia al alza en sus niveles de segregación para los cuatro grupos minoritarios analizados, especialmente, en el caso de los estudiantes con familias de menor nivel socioeconómico.

Krüger (2019) analiza la dinámica y la evolución de la segregación por nivel socioeconómico en 9 países de América Latina usando los datos de PISA del período 2000 y 2015 y los índices disimilitud, de información mutua y de aislamiento para el análisis. Dado su alto nivel de segregación, significativamente mayor al del resto de los países de la región, el Perú es calificado como una situación de hipersegregación.

Otro estudio más acotado a la realidad peruana es el de León y Collahua (2016), quienes identificaron, sobre la base de un metaanálisis de estudios realizados entre los años 2000 y 2014, que el nivel socioeconómico es una variable central para explicar el rendimiento de los estudiantes peruanos, tanto en el ámbito individual como en el escolar.

Cueto, León y Miranda (2016), encontraron - a partir de datos longitudinales del estudio de Young Lives - altos índices de segregación en las aulas peruanas y que la calidad de la escolaridad se asocia con la segregación de una manera que refuerza la desigualdad. Identificaron, además, que la segregación 
ocurre principalmente en los extremos (es decir, en las aulas que tienen muy pocos o la mayoría de los padres con educación secundaria o más).

Por su parte, Balarín $(2015,2016)$, y Balarín y Escudero (2018) estudiaron el impacto de los procesos de privatización sobre la segregación y la estratificación educativa, así como en la profundización de estas, tanto en el sector público como privado, explicado por los mecanismos generalizados y no gobernados de la elección de escuelas (school choice). Para las investigadoras, el uso extendido de la elección escolar tanto en el sistema educativo privado como en el estatal explica los patrones de segregación en las escuelas y que los altos niveles de segregación escolar que encuentran en Perú son el resultado de cómo se han establecido las dinámicas de elección educativa para las familias.

Esta investigación busca profundizar en el conocimiento de la segregación escolar por nivel socioeconómico en el total de escuelas de educación secundaria en Perú y sus regiones, estimando tanto en la dimensión de uniformidad como de aislamiento con una mirada específica en las escuelas situadas en contextos urbanos. Para ello, se utilizan los datos censales de la Evaluación Censal de Estudiantes (ECE) 2016 del Ministerio de Educación, lo que les permite mayor precisión a los resultados.

\section{Metodología}

Para cumplir con el propósito de este estudio, se realiza una explotación especial de las bases de datos de la ECE de Perú, desarrollada por la Oficina de Medición de la Calidad de los Aprendizajes (UMC) del Ministerio de Educación. La ECE tiene como uno de sus objetivos dar información sobre los logros de aprendizaje de los estudiantes peruanos en las áreas de Comunicación y Matemática $\left(2^{\circ}\right.$ grado de primaria, $4^{\circ}$ grado de primaria no bilingüe y $2^{\circ}$ grado de secundaria); Comunicación ( $4^{\circ}$ grado de primaria EIB); e Historia, Geografía y Economía ( $2^{\circ}$ grado de secundaria) (Ministerio de Educación, 2016). Además de estas pruebas, se aplican cuestionarios dirigidos a directores, docentes, estudiantes y padres de familia con el objetivo de explicar los resultados de las evaluaciones, y, de esta manera, generar evidencia sobre las características individuales y familiares de los estudiantes evaluados, así como de las condiciones y los procesos escolares que se encuentran asociados a su desempeño (Ministerio de Educación, 2018).

Este estudio se centra en los datos del 2016 de los estudiantes de $2^{\circ}$ grado de educación secundaria. La muestra utilizada es de un total de 489.161 estudiantes matriculados en 12.862 escuelas de educación secundaria de todo el país (Tabla 1). 
Tabla 1

Muestra y sus características

\begin{tabular}{|c|c|c|c|c|c|c|c|c|}
\hline & \multicolumn{3}{|c|}{ Total } & \multicolumn{5}{|c|}{ En entorno urbano } \\
\hline & $\begin{array}{l}\text { N. } \\
\text { estud. }\end{array}$ & $\begin{array}{c}\mathrm{N} . \\
\text { escuelas }\end{array}$ & $\begin{array}{c}\text { ISE } \\
\text { promedio }\end{array}$ & $\begin{array}{c}\mathrm{N} \\
\text { estud. }\end{array}$ & $\begin{array}{l}\% \text { est. } \\
\text { del total }\end{array}$ & $\begin{array}{c}\mathrm{N} \\
\text { escuelas }\end{array}$ & $\begin{array}{c}\% \text { esc } \\
\text { del total }\end{array}$ & $\begin{array}{c}\text { ISE } \\
\text { promedio }\end{array}$ \\
\hline Amazonas & 7.370 & 283 & $-0,9952$ & 5.168 & 70,12 & 132 & 46,64 & $-0,7351$ \\
\hline Áncash & 19.069 & 612 & $-0,2451$ & 15.410 & 80,81 & 373 & 60,95 & $-0,0179$ \\
\hline Apurímac & 8.450 & 304 & $-0,6399$ & 6.609 & 78,21 & 170 & 55,92 & $-0,4912$ \\
\hline Arequipa & 21.177 & 592 & 0,4650 & 20.830 & 98,36 & 572 & 96,62 & 0,4781 \\
\hline Ayacucho & 13.265 & 441 & $-0,5165$ & 10.818 & 81,55 & 250 & 56,69 & $-0,3547$ \\
\hline Cajamarca & 26.121 & 999 & $-0,7914$ & 17.762 & 68,00 & 422 & 42,24 & $-0,5030$ \\
\hline Callao & 14.316 & 272 & 0,5535 & 14.316 & 100,00 & 272 & 100,00 & 0,5535 \\
\hline Cusco & 24.872 & 627 & $-0,4167$ & 19.769 & 79,48 & 361 & 57,58 & $-0,2252$ \\
\hline Huancavelica & 8.604 & 351 & $-0,8474$ & 5.753 & 66,86 & 175 & 49,86 & $-0,6717$ \\
\hline Huánuco & 14.405 & 465 & $-0,6478$ & 11.254 & 78,13 & 236 & 50,75 & $-0,4373$ \\
\hline Ica & 12.729 & 242 & 0,4026 & 12.564 & 98,70 & 229 & 94,63 & 0,4112 \\
\hline Junín & 22.138 & 645 & $-0,1523$ & 19.494 & 88,06 & 449 & 69,61 & $-0,0121$ \\
\hline La Libertad & 28.041 & 783 & $-0,0371$ & 24.467 & 87,25 & 522 & 66,67 & 0,1433 \\
\hline Lambayeque & 19.605 & 408 & 0,0633 & 18.541 & 94,57 & 335 & 82,11 & 0,1365 \\
\hline Lima & 140.022 & 2.942 & 0,6301 & 138.675 & 99,04 & 2.854 & 97,01 & 0,6403 \\
\hline Loreto & 15.370 & 492 & $-0,6475$ & 12.955 & 84,29 & 234 & 47,56 & $-0,4578$ \\
\hline Madre de Dios & 2.382 & 63 & $-0,1561$ & 2.123 & 89,13 & 42 & 66,67 & $-0,0585$ \\
\hline Moquegua & 2.695 & 63 & 0,5115 & 2.625 & 97,40 & 55 & 87,30 & 0,5177 \\
\hline Pasco & 4.059 & 178 & $-0,3892$ & 3.195 & 78,71 & 92 & 51,69 & $-0,1499$ \\
\hline Piura & 31.441 & 741 & $-0,3144$ & 27.289 & 86,79 & 509 & 68,69 & $-0,1535$ \\
\hline Puno & 22.187 & 561 & $-0,3076$ & 18.551 & 83,61 & 333 & 59,36 & $-0,1821$ \\
\hline San Martín & 14.031 & 378 & $-0,5858$ & 12.222 & 87,11 & 250 & 66,14 & $-0,4551$ \\
\hline Tacna & 5.317 & 112 & 0,4454 & 5.140 & 96,67 & 100 & 89,29 & 0,4686 \\
\hline Tumbes & 3.578 & 78 & 0,0735 & 3.491 & 97,57 & 71 & 91,03 & 0,0988 \\
\hline Ucayali & 7.917 & 230 & $-0,3709$ & 7.066 & 89,25 & 115 & 50,00 & $-0,2477$ \\
\hline $\begin{array}{l}\text { Total/ } \\
\text { promedio }\end{array}$ & 489.161 & 12.862 & 0,0000 & 436.087 & 89,15 & 9.153 & 71,16 & 0,1537 \\
\hline
\end{tabular}

Fuente: Elaboración propia a partir de la base de datos ECE 2016 (Minedu-UMC 2016). 
La variable criterio utilizada para el estudio es el índice socioeconómico (ISE), que brinda información detallada para el posicionamiento socioeconómico relativo de cada individuo con respecto a toda la muestra. El ISE es estimado por el propio Ministerio de Educación (2017) a partir de variables sobre la educación de los padres, materiales de construcción de la vivienda, servicios básicos en el hogar, activos en el hogar y otros servicios en el hogar. La variable está tipificada (media 0 y desviación típica 1 ).

La segregación escolar se estimó con dos índices que miden dos dimensiones: el índice de Gorard estimó la dimensión de uniformidad; y el índice de aislamiento, la dimensión de exposición. El índice de segregación de Gorard (índice G) se define como la "proporción de estudiantes desfavorecidos que tendrían que intercambiar escuelas dentro del área del análisis, para que exista una distribución uniforme de este grupo entre la población" (Gorard y Smith, 2004, p. 19). Este índice es una variación del índice de Duncan y tiene una fácil interpretación, dado que es la única medida de asociación utilizable con más de dos casos que aparece completamente libre de la influencia, o los cambios en la población (Gorard y Taylor, 2000). Matemáticamente, se expresa de la siguiente forma:

$$
G=\frac{1}{2} \sum_{j=1}^{k}\left|\frac{X_{1 j}}{X_{1}}-\frac{T_{j}}{T}\right|
$$

En esta fórmula, para país, $\mathrm{x}_{1 \mathrm{i}}$ representa el número de alumnos del grupo minoritario en la escuela $\mathrm{i}$; $\mathrm{X}_{1}$ es el número total de estudiantes del grupo minoritario en todas las escuelas del país o la región; $\mathrm{T}_{\mathrm{i}}$ es el número total de alumnos en la escuela i; y T, el número total de alumnos en el país o la región.

El índice de aislamiento (índice A) propuesto por Lieberson (1981) mide la "probabilidad de que un estudiante del grupo minoritario se encuentre en su escuela con otro miembro de su mismo grupo" (Murillo, 2016, p. 43). De esta forma, cuanto mayor sea el aislamiento del grupo, menor será su exposición a los miembros del resto de grupos (Murillo, Duk y Martínez-Garrido, 2018). Es el índice más utilizado para medir la dimensión de exposición de la segregación escolar. El procedimiento de estimación es el siguiente:

$$
A=\sum_{j=1}^{k} \frac{X_{1 j}}{X_{1}} \frac{X_{1 j}}{T_{j}}
$$

En este, $\mathrm{x}_{1 \mathrm{i}}$ representa el número de alumnos del grupo minoritario en la escuela i; $\mathrm{X}_{1}$ es el número total de estudiantes minoritarios, respectivamente, en todas las escuelas del país o la región; $\mathrm{y}_{\mathrm{i}}$, el número total de alumnos en la escuela i. 
Para estimar ambos índices, se ha optado por definir cuatro grupos minoritarios: los percentiles 10 (P10) y 90 (P90), y los cuartiles 1 (Q1) y 4 (Q4); es decir, el 10\% (P) y el 25\% (Q) de los estudiantes con familias de menor (P10 y Q1) y de mayor (P90 y Q4) nivel socioeconómico (ISE), respectivamente. También, se estimó la puntuación promedio de la segregación para los cuatro colectivos. Cada grupo minoritario se compara con el grupo mayoritario, que correspondería al resto de estudiantes.

\section{Resultados}

Un primer hallazgo del estudio es que la magnitud de la segregación escolar por nivel socioeconómico en educación secundaria para el Perú es alta, tanto en su dimensión de uniformidad (índice de Gorard 0,54) como en su dimensión de exposición (índice de aislamiento de 0,50) para el total de escuelas del país. Un segundo hallazgo es que la segregación es mayor para los estudiantes de secundaria con familias de menor nivel socioeconómico. Así, la magnitud de la segregación en los estudiantes de familias de menos ISE es de 0,64 (P10, índice de Gorard) y 0,62 (Q1, índice de aislamiento).

Estos resultados, sin embargo, adquieren valores diversos al estimar la segregación para cada una de las veinticinco regiones del país, lo que constituye el tercer hallazgo de este estudio. Según la dimensión de uniformidad, las magnitudes van desde el 0,36 hasta el 0,54 (índice de Gorard) y en la dimensión de exposición, de 0,33 a 0,51 (índice de aislamiento). Así, se identifica en la Tabla 2 que el orden de las regiones varía en función del grupo estudiado, según el índice de Gorard:

- Para el $10 \%$ de estudiantes de familias con menor nivel socioeconómico (P10), las regiones con segregación superior a 0,6 (que puede ser considerada hipersegregación) son Pasco $(0,65)$, La Libertad $(0,65)$ y Loreto $(0,64)$. Las regiones con menor segregación son Ica $(0,37)$, Tumbes $(0,39)$ y Huancavelica $(0,41)$.

- Para el 25\% con menor nivel socioeconómico (Q1), las 3 regiones con la segregación más alta son La Libertad $(0,50)$, Loreto $(0,48)$ y Piura $(0,47)$. Aquellas que tienen la segregación más baja son las mismas y en el mismo orden que en el caso del P10: Ica y Tumbes (ambas con 0,28) y Huancavelica $(0,31)$.

- Para el 25\% con mayor nivel socioeconómico (Q4), la mayor segregación se da en las regiones de Cajamarca $(0,51)$, Huánuco $(0,50)$ y Áncash $(0,47)$. Las de menor segregación son Madre de Dios $(0,31)$, Moquegua $(0,33)$ e Ica $(0,35)$.

- Por último, para el $10 \%$ de estudiantes de familias con mayor nivel socioeconómico (P90), las regiones con mayor segregación son Cajamarca $(0,64)$, Huánuco $(0,62)$ y Apurímac $(0,60)$, y las tres de menor segregación escolar son Moquegua $(0,38)$, Madre de Dios $(0,43)$ y Tumbes $(0,45)$. 
Tabla 2

Segregación escolar por nivel socioeconómico en Perú y sus regiones Índice de Gorard para P10, Q1, Q4, P10 como grupos minoritarios, y promedio

\begin{tabular}{lccccc}
\hline & $\mathrm{P} 10$ & $\mathrm{Q} 1$ & $\mathrm{Q} 4$ & $\mathrm{P} 90$ & Promedio \\
\hline Perú & 0,6370 & 0,5072 & 0,4583 & 0,5743 & 0,5442 \\
Amazonas & 0,5913 & 0,4493 & 0,4374 & 0,5829 & 0,5152 \\
Áncash & 0,5549 & 0,4574 & 0,4676 & 0,5778 & 0,5144 \\
Apurímac & 0,4322 & 0,3282 & 0,4284 & 0,6000 & 0,4472 \\
Arequipa & 0,5253 & 0,4084 & 0,4209 & 0,5528 & 0,4768 \\
Ayacucho & 0,4979 & 0,3886 & 0,4319 & 0,5993 & 0,4794 \\
Cajamarca & 0,5157 & 0,3902 & 0,5086 & 0,6403 & 0,5137 \\
Callao & 0,5474 & 0,4227 & 0,3647 & 0,4666 & 0,4503 \\
Cusco & 0,5291 & 0,4159 & 0,4639 & 0,5932 & 0,5005 \\
Huancavelica & 0,4149 & 0,3073 & 0,3818 & 0,5816 & 0,4214 \\
Huánuco & 0,4770 & 0,3795 & 0,4984 & 0,6204 & 0,4938 \\
Ica & 0,3666 & 0,2806 & 0,3455 & 0,4587 & 0,3628 \\
Junín & 0,5916 & 0,4197 & 0,4189 & 0,5531 & 0,4958 \\
La Libertad & 0,6490 & 0,5018 & 0,4381 & 0,5718 & 0,5402 \\
Lambayeque & 0,5730 & 0,4323 & 0,4308 & 0,5623 & 0,4996 \\
Lima & 0,4883 & 0,3647 & 0,3806 & 0,5215 & 0,4388 \\
Loreto & 0,6400 & 0,4831 & 0,4549 & 0,5781 & 0,5390 \\
Madre de Dios & 0,4629 & 0,3449 & 0,3092 & 0,4250 & 0,3855 \\
Moquegua & 0,4439 & 0,3459 & 0,3278 & 0,3813 & 0,3747 \\
Pasco & 0,6492 & 0,4344 & 0,3758 & 0,4829 & 0,4856 \\
Piura & 0,5972 & 0,4722 & 0,4457 & 0,5962 & 0,5278 \\
Puno & 0,4611 & 0,3738 & 0,4037 & 0,5399 & 0,4446 \\
San Martín & 0,5215 & 0,4109 & 0,4282 & 0,5493 & 0,4775 \\
Tacna & 0,4371 & 0,3498 & 0,3669 & 0,4899 & 0,4109 \\
Tumbes & 0,3931 & 0,2833 & 0,3469 & 0,4516 & 0,3687 \\
Ucayali & 0,5719 & 0,3773 & 0,3750 & 0,5130 & 0,4593 \\
\hline & & & & &
\end{tabular}

Fuente: Elaboración propia a partir de la base de datos ECE 2016 (Minedu-UMC 2016). 
En este grupo de regiones, se evidencia que, si bien los índices de segregación son altos en los cuatro grupos, hay regiones con muy alta segregación en los extremos (P10 y P90), con tendencia a la hipersegración en el grupo con menor nivel socioeconómico (P10). Destacan La Libertad y Loreto como las regiones con las escuelas más segregadas del Perú en los grupos con menor nivel socioeconómico (P10 y Q1), mientras que Cajamarca es la más segregada para los grupos con mayor nivel socioeconómico (Q4 y P90).

Los resultados encontrados en su dimensión de exposición, según el índice de aislamiento (Tabla 3), evidencian una magnitud de segregación menor en los grupos P10 y P90 (10\% de estudiantes con menor y mayor ISE) que en los grupos Q1 y Q4 (25\% de estudiantes con menor y mayor ISE). Estos resultados son lo esperado en este índice, dada la concepción del mismo. A continuación, se identifican las diferencias entre las regiones:

- Al considerar el P10 como grupo minoritario, las regiones con mayor segregación son Pasco $(0,49)$, Loreto $(0,47)$ y Ucayali $(0,46)$. Por el contrario, las regiones de Ica $(0,22)$, Huancavelica $(0,23)$ y Tumbes $(0,23)$ son las que presentan una menor segregación.

- Para el Q1 como grupo minoritario, La Libertad $(0,63)$, Loreto $(0,60)$ y Piura $(0,58)$ son las tres regiones con una mayor segregación escolar; e Ica $(0,38)$, Tumbes $(0,39)$ y Huancavelica $(0,39)$, las de menor.

- Para el caso del 25\% de estudiantes con familias de mayor nivel socioeconómico $(\mathrm{Q} 4)$, las regiones más segregadas son Cajamarca $(0,62)$, Huánuco $(0,61)$ y Cusco $(0,58)$, mientras que Madre de Dios $(0,44)$, Moquegua $(0,44)$ e Ica $(0,45)$ son las que presentan menor segregación.

- Por último, al considerar el P90 como grupo minoritario, las regiones con mayor segregación son Cajamarca $(0,47)$, Ayacucho $(0,47)$ y Cusco $(0,44)$; y las que tiene menor segregación Moquegua $(0,24)$, Madre de Dios $(0,25)$ y Callao $(0,26)$. 
Tabla 3

Segregación escolar por nivel socioeconómico en Perú y sus regiones Índice de aislamiento para P10, Q1, Q4, P10 como grupos minoritarios, y promedio

\begin{tabular}{|c|c|c|c|c|c|}
\hline & P10 & Q1 & Q4 & P90 & $\begin{array}{c}\text { Prome- } \\
\text { dio }\end{array}$ \\
\hline Perú & 0,4442 & 0,6231 & 0,5614 & 0,3848 & 0,5034 \\
\hline Amazonas & 0,3738 & 0,5481 & 0,5252 & 0,3610 & 0,4521 \\
\hline Áncash & 0,3361 & 0,5466 & 0,5746 & 0,3643 & 0,4554 \\
\hline Apurímac & 0,2459 & 0,4163 & 0,5375 & 0,4147 & 0,4036 \\
\hline Arequipa & 0,3277 & 0,5019 & 0,5180 & 0,3467 & 0,4236 \\
\hline Ayacucho & 0,3163 & 0,4847 & 0,5487 & 0,4645 & 0,4535 \\
\hline Cajamarca & 0,2936 & 0,4693 & 0,6236 & 0,4718 & 0,4646 \\
\hline Callao & 0,2885 & 0,4980 & 0,4539 & 0,2545 & 0,3737 \\
\hline Cusco & 0,3216 & 0,4994 & 0,5757 & 0,4438 & 0,4601 \\
\hline Huancavelica & 0,2283 & 0,3927 & 0,4930 & 0,4429 & 0,3893 \\
\hline Huánuco & 0,2948 & 0,4648 & 0,6114 & 0,4434 & 0,4536 \\
\hline Ica & 0,2215 & 0,3789 & 0,4508 & 0,2771 & 0,3321 \\
\hline Junín & 0,4561 & 0,5436 & 0,5269 & 0,3683 & 0,4737 \\
\hline La Libertad & 0,4447 & 0,6293 & 0,5454 & 0,3796 & 0,4998 \\
\hline Lambayeque & 0,3965 & 0,5459 & 0,5356 & 0,3551 & 0,4583 \\
\hline Lima & 0,2873 & 0,4470 & 0,4781 & 0,3304 & 0,3857 \\
\hline Loreto & 0,4701 & 0,6020 & 0,5562 & 0,4121 & 0,5101 \\
\hline Madre de Dios & 0,3003 & 0,4425 & 0,4358 & 0,2527 & 0,3578 \\
\hline Moquegua & 0,2599 & 0,4324 & 0,4397 & 0,2379 & 0,3425 \\
\hline Pasco & 0,4925 & 0,5519 & 0,4647 & 0,2789 & 0,4470 \\
\hline Piura & 0,3966 & 0,5781 & 0,5624 & 0,4197 & 0,4892 \\
\hline Puno & 0,2621 & 0,4535 & 0,5161 & 0,3724 & 0,4010 \\
\hline San Martín & 0,3183 & 0,4982 & 0,5326 & 0,3825 & 0,4329 \\
\hline Tacna & 0,2937 & 0,4502 & 0,4676 & 0,2953 & 0,3767 \\
\hline Tumbes & 0,2288 & 0,3878 & 0,4609 & 0,2884 & 0,3415 \\
\hline Ucayali & 0,4643 & 0,5028 & 0,4708 & 0,2961 & 0,4335 \\
\hline
\end{tabular}

Fuente: Elaboración propia a partir de la base de datos ECE 2016 (Minedu-UMC 2016). 
En este caso, se evidencia que, si bien los resultados son similares al análisis anterior, en los grupos de menor nivel socioeconómico (P10 y Q1), Loreto $(0,51)$ es la región con mayor nivel de segregación, mientras que Ica $(0,33)$, Tumbes $(0,34)$ y Huancavelica $(0,39)$ son las regiones con menor nivel de segregación. En los grupos de mayor nivel socioeconómico (Q4 y P90), Cajamarca $(0,47)$ y Cusco $(0,46)$ son las regiones con mayor segregación, mientras que Moquegua $(0,34)$ es la menos segregada.

Estos resultados podrían ser explicados por la segregación residencial, a partir de las diferencias de las escuelas secundarias ubicadas en zonas urbanas y rurales. En esa línea, Murillo y Martínez-Garrido (2017a) proponen estudiar la segregación escolar en las zonas urbanas, dado que la segregación residencial se produce esencialmente en zonas rurales. Es decir, si en un determinado lugar geográfico hay una sola institución educativa, esta necesariamente recogerá a todos los estudiantes de esa zona y, si en esa zona, hay una alta concentración de familias de determinado nivel socioeconómico, la segregación será alta.

Dada las posibilidades, mínimas o nulas, que tienen las familias de zonas rurales de elegir una escuela secundaria, la segregación escolar en esas zonas podría explicarse esencialmente la segregación residencial, por lo que los datos globales podrían estar influidos por el número de escuelas en zonas rurales. En ese sentido, un análisis más profundo de la segregación escolar puede darse a partir del análisis exclusivo de las escuelas secundarias que se encuentran ubicadas en zonas urbanas. En las tablas 4 y 5 , se muestran los resultados de estimar los índices de segregación (Gorard y Aislamiento) solo en las escuelas secundarias urbanas del país y sus regiones. Como un cuarto hallazgo, se identifica que los resultados anteriores se matizan, pero las diferencias se mantienen.

Según el índice de Gorard (Tabla 4), el promedio de la segregación escolar socioeconómica para las escuelas de secundaria urbanas del país, en su dimensión de igualdad o uniformidad, es de 0,51 ( 0,54 para el total), manteniéndose una variabilidad en los promedios de las regiones (de 0,35 a 0,49 ). El promedio en las regiones se distribuye de la siguiente manera, según los grupos minoritarios:

- Para el $10 \%$ de estudiantes de familias con menos nivel socioeconómico (P10), la región con hipersegregación (más de 0,6$)$ es Amazonas $(0,61$ ). Las regiones de La Libertad $(0,57)$, Loreto $(0,56)$, Callao $(0,55)$, Piura $(0,54)$, Lambayeque $(0,54)$, Arequipa $(0,52)$, Áncash $(0,51)$, Cusco $(0,50)$ y Cajamarca $(0,50)$ tienen una segregación muy alta.

- Para el 25\% con menos nivel socioeconómico (Q1), las regiones con los índices de segregación altos son La Libertad $(0,43)$, Loreto $(0,43)$, Áncash $(0,43)$, Callao $(0,42)$, Piura $(0,42)$, Amazonas $(0,42)$, Cajamarca $(0,41)$, Arequipa $(0,40)$ y Lambayeque $(0,40)$.

- Para el 25\% con mayor nivel socioeconómico (Q4), las regiones con niveles de segregación altos — de más de 0,40 -, son Huánuco $(0,47)$, Cajamarca $(0,46)$, Piura $(0,43)$, Cusco $(0,43)$, Loreto $(0,43)$, Lambayeque $(0,43)$, Áncash $(0,42)$, Arequipa $(0,42)$, Apurímac $(0,42)$, La Libertad $(0,41)$, Ayacucho $(0,41)$, San Martín $(0,41)$ y Junín $(0,40)$.

20 I Para el $10 \%$ de estudiantes de familias con mayor nivel socioeconómico (P90), las regiones con niveles de segregación muy altos — es decir, más de 
0,5- son Cajamarca $(0,59)$, Huánuco $(0,58)$, Ayacucho $(0,58)$, Piura $(0,58)$, Huancavelica $(0,58)$, Apurímac $(0,56)$, Cusco $(0,56)$, Lambayeque $(0,55)$, Arequipa $(0,55)$, La Libertad $(0,55)$, Loreto $(0,54)$, Áncash $(0,54)$, San Martín $(0,53)$, Junín $(0,53)$, Puno $(0,53)$, Amazonas $(0,53)$ y Lima $(0,52)$.

Tabla 4

Segregación escolar por nivel socioeconómico en Perú y sus regiones para escuelas urbanas

Índice de Gorard para P10, Q1, Q4, P10 como grupos minoritarios, y promedio

\begin{tabular}{lccccc}
\hline & $\mathrm{P} 10$ & $\mathrm{Q} 1$ & $\mathrm{Q} 4$ & $\mathrm{P} 90$ & Promedio \\
\hline Perú & 0,5943 & 0,4635 & 0,4360 & 0,5546 & 0,5121 \\
Amazonas & 0,6066 & 0,4145 & 0,3814 & 0,5276 & 0,4825 \\
Áncash & 0,5147 & 0,4271 & 0,4235 & 0,5358 & 0,4752 \\
Apurímac & 0,3723 & 0,3151 & 0,4168 & 0,5664 & 0,4177 \\
Arequipa & 0,5173 & 0,4033 & 0,4193 & 0,5489 & 0,4722 \\
Ayacucho & 0,4480 & 0,3567 & 0,4107 & 0,5813 & 0,4492 \\
Cajamarca & 0,5013 & 0,4089 & 0,4576 & 0,5860 & 0,4884 \\
Callao & 0,5474 & 0,4227 & 0,3647 & 0,4666 & 0,4503 \\
Cusco & 0,5023 & 0,3963 & 0,4302 & 0,5593 & 0,4720 \\
Huancavelica & 0,3649 & 0,2950 & 0,3784 & 0,5773 & 0,4039 \\
Huánuco & 0,4316 & 0,3762 & 0,4684 & 0,5820 & 0,4646 \\
Ica & 0,3607 & 0,2784 & 0,3458 & 0,4567 & 0,3604 \\
Junín & 0,4959 & 0,3717 & 0,4023 & 0,5327 & 0,4506 \\
La Libertad & 0,5704 & 0,4276 & 0,4117 & 0,5487 & 0,4896 \\
Lambayeque & 0,5373 & 0,4014 & 0,4254 & 0,5500 & 0,4785 \\
Lima & 0,4790 & 0,3602 & 0,3790 & 0,5201 & 0,4346 \\
Loreto & 0,5587 & 0,4273 & 0,4273 & 0,5441 & 0,4894 \\
Madre de Dios & 0,4231 & 0,2738 & 0,3009 & 0,3920 & 0,3475 \\
Moquegua & 0,4279 & 0,3352 & 0,3226 & 0,3639 & 0,3624 \\
Pasco & 0,4735 & 0,3387 & 0,3021 & 0,4309 & 0,3863 \\
Piura & 0,5382 & 0,4169 & 0,4318 & 0,5790 & 0,4915 \\
Puno & 0,4417 & 0,3472 & 0,3757 & 0,5301 & 0,4237 \\
San Martín & 0,4673 & 0,3743 & 0,4064 & 0,5341 & 0,4455 \\
Tacna & 0,4179 & 0,3368 & 0,3599 & 0,4817 & 0,3991 \\
Tumbes & 0,3746 & 0,2753 & 0,3386 & 0,4439 & 0,3581 \\
Ucayali & 0,3976 & 0,3133 & 0,3598 & 0,4817 & 0,3881 \\
\hline & & & & &
\end{tabular}

Fuente: Elaboración propia a partir de la base de datos ECE 2016 (Minedu-UMC 2016). 
En este análisis, en concordancia con el análisis anterior, se identifica que, si bien los índices de segregación son altos en los cuatro grupos, hay regiones con segregación muy alta en los extremos (P10 y P90) a nivel de escuelas urbanas, con tendencia a la hipersegración en el grupo de menor nivel socioeconómico (P10). Destacan Amazonas y Callao como las regiones más segregadas en los grupos con menor nivel socioeconómico (P10 y Q1), mientras que Piura, La Libertad, Loreto, Cajamarca, Lambayeque, Áncash y Arequipa lo hacen en los cuatro grupos. Las regiones más segregadas en los grupos con mayor nivel socioeconómico (Q4 y P90) son Ayacucho, San Martín y Apurímac.

En su dimensión de exposición, los resultados encontrados en la segregación escolar socioeconómica, según el índice de aislamiento (Tabla 5), para las escuelas de secundaria urbanas disminuye a 0,36 (0,50 para el total), lo cual muestra una variabilidad entre las regiones de 0,32 a 0,45. El promedio en las regiones se distribuye de la siguiente manera, según los grupos minoritarios:

- $\mathrm{Al}$ considerar el P10 como grupo minoritario, las tres regiones con mayor segregación son Amazonas $(0,42)$, La Libertad $(0,40)$ y Loreto $(0,38)$; y Huancavelica $(0,19)$, Tumbes $(0,20)$ y Apurímac $(0,20)$, las de menor.

- Para el Q1 como grupo minoritario, las regiones con mayor segregación son La Libertad $(0,54)$, Loreto $(0,52)$ y Piura $(0,52)$. Las regiones de Tumbes $(0,37)$, Ica $(0,38)$ y Huancavelica $(0,39)$ son las de menor segregación.

- Para el 25\% de estudiantes de familias con mayor nivel socioeconómico (Q4), las regiones con mayor segregación son Huánuco $(0,58)$, Cajamarca $(0,57)$ y Cusco $(0,55)$. En el extremo opuesto, las regiones de Pasco $(0,41)$, Madre de Dios $(0,42)$ y Moquegua $(0,44)$ son las de menor segregación.

- Para finalizar, si se considera el P90 de los estudiantes como grupo minoritario, las tres regiones con mayor segregación son Ayacucho $(0,45)$, Huancavelica $(0,42)$ y Cajamarca $(0,42)$; y las tres regiones con menor son, en este caso, Madre de Dios $(0,23)$, Moquegua $(0,23)$ y Callao $(0,26)$. 
Tabla 5

Segregación escolar por nivel socioeconómico en Perú y sus regiones para escuelas urbanas Índice de aislamiento para P10, Q1, Q4, P10 como grupos minoritarios, y promedio

\begin{tabular}{lccccc}
\hline & $\mathrm{P} 10$ & $\mathrm{Q} 1$ & $\mathrm{Q} 4$ & $\mathrm{P} 90$ & Promedio \\
\hline Perú & 0,4101 & 0,3627 & 0,3156 & 0,3583 & 0,3617 \\
Amazonas & 0,4159 & 0,5166 & 0,4702 & 0,3088 & 0,4279 \\
Áncash & 0,2817 & 0,5095 & 0,5187 & 0,3239 & 0,4084 \\
Apurímac & 0,1987 & 0,3990 & 0,5240 & 0,3735 & 0,3738 \\
Arequipa & 0,3115 & 0,4965 & 0,5154 & 0,3441 & 0,4169 \\
Ayacucho & 0,2490 & 0,4411 & 0,5325 & 0,4466 & 0,4173 \\
Cajamarca & 0,2767 & 0,4830 & 0,5737 & 0,4158 & 0,4373 \\
Callao & 0,2885 & 0,4980 & 0,4539 & 0,2545 & 0,3737 \\
Cusco & 0,2786 & 0,4704 & 0,5516 & 0,3994 & 0,4250 \\
Huancavelica & 0,1895 & 0,3845 & 0,5037 & 0,4232 & 0,3752 \\
Huánuco & 0,2419 & 0,4529 & 0,5791 & 0,4090 & 0,4207 \\
Ica & 0,2081 & 0,3745 & 0,4500 & 0,2745 & 0,3268 \\
Junín & 0,3231 & 0,4676 & 0,5072 & 0,3474 & 0,4113 \\
La Libertad & 0,4028 & 0,5402 & 0,5148 & 0,3574 & 0,4538 \\
Lambayeque & 0,3416 & 0,5009 & 0,5257 & 0,3461 & 0,4286 \\
Lima & 0,2685 & 0,4391 & 0,4766 & 0,3304 & 0,3787 \\
Loreto & 0,3834 & 0,5222 & 0,5245 & 0,3807 & 0,4527 \\
Madre de Dios & 0,2493 & 0,3856 & 0,4145 & 0,2283 & 0,3194 \\
Moquegua & 0,2325 & 0,4146 & 0,4345 & 0,2320 & 0,3284 \\
Pasco & 0,2468 & 0,4201 & 0,4077 & 0,2569 & 0,3329 \\
Piura & 0,3323 & 0,5172 & 0,5439 & 0,4000 & 0,4484 \\
Puno & 0,2430 & 0,4296 & 0,4932 & 0,3573 & 0,3808 \\
San Martín & 0,2674 & 0,4571 & 0,5112 & 0,3670 & 0,4007 \\
Tacna & 0,2607 & 0,4270 & 0,4624 & 0,2951 & 0,3613 \\
Tumbes & 0,1962 & 0,3671 & 0,4525 & 0,2856 & 0,3254 \\
Ucayali & 0,2474 & 0,4070 & 0,4536 & 0,2761 & 0,3460 \\
\hline & & & & &
\end{tabular}

Fuente: Elaboración propia a partir de la base de datos ECE 2016 (Minedu-UMC 2016). 
Para este análisis, las regiones de La Libertad y Loreto son las que cuentan con escuelas secundaria con una alta segregación en los grupos de menor nivel socioeconómico. En este mismo grupo, Huancavelica y Tumbes presentan la menor segregación. Madre de Dios y Moquegua son las dos regiones que presentan escuelas urbanas de secundaria con menor segregación en los grupos de nivel socioeconómico más alto.

\section{Discusión y conclusiones}

Este estudio ha estimado la magnitud de la segregación escolar por nivel socioeconómico en educación secundaria en Perú y en sus veinticinco regiones, con una mirada específica en las escuelas urbanas. Así, se ha encontrado altos niveles de segregación escolar en su dimensión de uniformidad (índice de Gorard de 0,54 para todas las escuelas y 0,51 solo urbanas). Estos resultados dan cuenta de que cinco de cada diez estudiantes deberían cambiarse de escuela para lograr un reparto igual a la presencia de los estudiantes de su mismo grupo en la población total. En cuanto a su dimensión de exposición, los datos evidencian que la probabilidad de que un estudiante se encuentre en su escuela con otro estudiante de su mismo grupo es del 50\% en cualquier escuela secundaria del país, y del 36\% en las escuelas solo urbanas (índice de aislamiento de 0,50 para todas las escuelas y 0,36 solo urbanas). En este caso, se podría suponer que el índice de aislamiento es un poco menor en las escuelas urbanas por la migración de estudiantes del campo a la ciudad para seguir estudios secundarios. Al respecto, sería interesante comprobar esta hipótesis y profundizar en los datos según provincias y regiones.

Las cifras encontradas para el conjunto de las escuelas de secundarias de Perú son análogas a las obtenidas por Murillo, Duk y Martínez-Garrido (2018), utilizando PISA en su versión 2015. Los índices de Gorard que arroja este estudio son de 0,60 para el $10 \%$ de los estudiantes de familias con menos nivel socioeconómico (P10), cerca de los 0,64 hallados en este trabajo; de 0,48 para Q1, frente a 0,51; de 0,41 para el Q4, frente a los 0,46; y de 0,55 para P90, frente a 0,57. En el caso del índice de aislamiento, Murillo, Duk y Martínez-Garrido encontraron una segregación de 0,37 para el P10 frente a 0,44 encontrados en este estudio; de 0,57 para Q1, frente a 0,62; de 0,50 para Q4, frente a 0,56; y de 0,55 para P90, frente a 0,39 . Esas diferencias entre 0,02 y 0,05 puntos para el índice de Gorard y 0,05 y 0,16 para el índice de aislamiento reflejan resultados que guardan coherencia con los hallazgos de este trabajo. Las diferencias pueden deberse a que la variable criterio es distinta. En PISA, se usa el índice de nivel socioeconómico y cultural de las familias de los estudiantes (ESCS), en contraste con el índice socioeconómico (ISE) utilizada en la ECE. También, cabe considerar que los datos de PISA son del 2015, mientras que los datos de la ECE son del año 2016. Además, el grado y su correlación con los grupos de edad evaluados es diferente en cada caso: ECE evalúa a estudiantes de segundo de secundaria, que tienen catorce años, mientras que PISA evalúa adolescentes de tercero de secundaria, que tienen quince años. 
Otro de los hallazgos de este estudio se relaciona con las diferencias significativas en la magnitud de la segregación entre las veinticinco regiones, lo que evidencia que la tendencia a la segregación es mayor para el total de escuelas del país (urbanas y rurales) y en los grupos de menor nivel socioeconómico. Estos resultados confirman la evidencia encontrada en otros estudios en cuanto el alto nivel de segregación escolar de los estudiantes peruanos en la secundaria (Benavides, León y Etesse, 2014; Krüger, 2019; Murillo, 2016; Murillo y Martínez-Garrido, 2017a; Murillo, Duk y Martínez-Garrido, 2018; Vázquez, 2012).

Los resultados que aquí se presentan generan más preguntas que respuestas, por lo que es necesario seguir profundizando y explorando las diferentes dimensiones que suponen la escolarización secundaria en el país. Bonal y Bellei (2018) explican que algunos de los factores que inducen los procesos de segregación escolar son externos a los sistemas educativos, por lo que requieren de una acción política más allá de la política educativa. En este sentido, es preciso identificar la segregación residencial como uno de los factores que explican estos resultados, tanto a nivel urbano-rural (total de escuelas), como la misma organización residencial en las zonas urbanas de todo el país (distritos residenciales, zonas urbano-marginales, etc.).

Murillo y Martínez-Garrido (2017a) señalan, además, que otras causas de la segregación escolar pueden evidenciarse en las características de los sistemas educativos o en políticas educativas específicas que pueden favorecer la polarización y una distribución desequilibrada de los estudiantes desfavorecidos o altamente privilegiados. Así, por ejemplo, frente a una diversidad en la oferta de servicios para el caso peruano, podríamos identificar oferta urbana y rural, así como pública y privada, diferenciada en cantidad y calidad. En la oferta pública de secundaria, se pueden encontrar los colegios de alto rendimiento, la jornada escolar completa, la jornada regular, así como las formas de atención diversificada en la secundaria rural (centros de alternancia, secundaria tutorial y residencias). Por su parte, en la oferta privada, se pueden identificar colegios que ofrecen bachillerato internacional, colegios preuniversitarios, colegios religiosos y laicos, alternativos y tradicionales, por nombrar algunos. Siguiendo a Orfield y Lee (2005), cabría preguntarse si se estaría utilizando la existencia de estas escuelas y su diversidad en la oferta para afirmar que podemos tener éxito educativo en general dentro de un contexto que profundiza la segregación.

En paralelo, las desigualdades en la calidad de la educación entre las escuelas públicas y privadas, y los altos niveles de segregación urbana entre las clases altas configuran escenarios específicos de extrema segregación educativa entre los ricos y los pobres (Bonal y Bellei, 2018). En el caso de Perú, la expansión de la educación privada ha sido un asunto que ha venido siendo investigado en los últimos años, particularmente, en un contexto con marcadas desigualdades educativas (Bello y Villarán, 2004; de Belaunde, 2011; Guadalupe et al., 2017; Rivas, 2015). Así, se profundiza, principalmente, sobre las razones por las que las familias optan por la educación privada, en línea con los 
enfoques de school choice (Balarín 2015; Sanz 2014, 2015) y las consecuencias de un mercado educativo privado, muy heterogéneo y altamente desregulado, en la alta segregación educativa que existe en el país (Balarín 2016; Balarín y Escudero, 2018; Cuenca, 2013; Cuenca, Reátegui y Oré, 2019). Se considera que esta es una línea de investigación que debe seguir desarrollándose desde la mirada de la segregación.

Asimismo, es necesario profundizar en la relación entre rendimiento y segregación escolar. Las evidencias apuntan a que la segregación afecta el aprendizaje de los estudiantes menos favorecidos (Cueto et al., 2016; Dupriez y Dumay, 2006; Gorard, 2014), lo que refuerza la desigualdad. Estudios como este, que utilizan datos censales, pueden llegar a desarrollar mapas regionales que identifiquen las provincias más segregadas, y, de esta manera, poder priorizar el diseño y la implementación de políticas que mejoren las condiciones y reduzcan los niveles de segregación, según los diversos contextos regionales. En algunos casos, estas políticas implicarán decisiones sectoriales, propiamente educativas, pedagógicas y de gestión. En otros, se requerirá de una acción intersectorial e intergubernamental, que apunte a problemáticas sociales más complejas. De cualquier manera, es un primer insumo y son datos muy potentes para seguir indagando bajo esta línea temática.

De esta manera, estos resultados aportan a la reflexión acerca de cómo la segregación escolar es un gran obstáculo para alcanzar la equidad, porque incide claramente en la desigualdad social (Murillo, 2016). La secundaria, como parte de la trayectoria escolar, es el nivel en que muchos de los aprendizajes se consolidan, en la medida que es una etapa clave para la formación, así como una oportunidad para generar mayor cohesión social y consolidar una ciudadanía activa, comprometida con el bien común y la justicia social (Organización de las Naciones Unidas, 2018). Se trata, entonces, de garantizar la igualdad de oportunidades entre todos los estudiantes, así como de construir una sociedad más justa, equitativa e inclusiva a través de un sistema educativo de análogas características. 


\section{Referencias bibliográficas}

Balarín, M. (2015). Las múltiples formas y efectos de la participación del sector privado en la educación. Balance de investigación. Lima: Forge-Grade.

(2016). La privatización por defecto y el surgimiento de las escuelas privadas de bajo costo en el Perú. ¿Cuáles son sus consecuencias? Revista de la Asociación de Sociología de la Educación, 9(2), 181-196. https://doi. org/10.7203/RASE.9.2.8414

Balarín, M. y Escudero, A. (2018). The ungoverned education market and the deepening of socio-economic school segregation in Peru. En Bonal, X. Y Bellei, C. (Ed.), Understanding School Segregation. Patterns, Causes and Consequences of Spatial Inequalities in Education (pp. 179-199). Londres: Bloomsbury Academic.

Banco Mundial. (2001). Peru. La educación en una encrucijada. Retos y oportunidades para el siglo XXI. Lima: Banco Mundial.

Bellei, C. (2007). Expansión de la educación privada y mejoramiento de la educación en Chile. Evaluación a partir de la evidencia. Pensamiento Educativo, 40(1), 1-21.

(2013). El estudio de la segregación económica y académica de la educación chilena. Estudios Pedagógicos, 39(1), 325-345.

Bellei, C., Contreras, M., Canales, M. y Orellana, V. (2018). The Production of Socio-economic Segregation in Chilean Education: School Choice, Social Class and Market Dynamics En Bonal, X. y Bellei, C. (Ed.), Understanding School Segregation. Patterns, Causes and Consequences of Spatial Inequalities in Education (pp. 221-240). Londres: Bloomsbury Academic.

Bello, M. y Villarán, V. (2004). Educación, reformas y equidad en los países de los andes y cono sur: Dos escenarios en el Perú. Buenos Aires: IIPE-Unesco.

Benavides, M., León, J. y Etesse, M. (2014). Desigualdades educativas y segregación en el sistema educativo peruano: una mirada comparativa de las pruebas PISA 2000 y 2009. Lima: Grade.

Bonal, X. (Ed.). (2006). Globalización, educación y pobreza en América Latina ¿Hacia una nueva agenda política? Barcelona: Fundación CIDOB.

Bonal, X. y Bellei, C. (Eds.). (2018). Understanding school segregation. Patterns, causes and consequences of spatial inequalities in education. Londres: Bloomsbury Academic.

Cárdenas Denham, S. (2011). Escuelas de doble turno en México: Una estimación de diferencias asociadas con su implementación. Revista Mexicana de Investigación Educativa, 16(50), 801-827.

Cuenca, R. (2013). La escuela pública en Lima Metropolitana. ¿Una institución en extinción? Revista Peruana de Investigación Educativa, 5, 73-98. 
Cuenca, R. (Ed.) (2015). La educación universitaria en el Perú: Democracia, expansión y desigualdades. Lima: Instituto de Estudios Peruanos.

Cuenca, R., Carrillo, S., De Los Ríos, C., Reátegui, L. y Ortiz, G. (2017). La calidad y equidad de la educación secundaria en el Perú. Lima: Instituto de Estudios Peruanos.

Cuenca, R., Reátegui, L. y Oré, S. (2019). Itinerarios de la educación privada en el Perú. Educação \& Sociedade 40(2). https://doi.org/10.1590/es010173302019192469.

Cueto, S. (2004). Factores predictivos del rendimiento escolar, deserción e ingreso a educación secundaria en una muestra de estudiantes de zonas rurales del Peru. Education Policy Analysis Archives, 12(35).

Cueto, S., León, J. y Miranda, A. (2016) Classroom composition and its association with students' achievement and socioemotional characteristics in Peru. Assessment in Education: Principles, Policy \& Practice, 23(1). https://doi.org/126-148. 10.1080/0969594X.2015.1105783

Cueto, S., León, J. y Muñoz, I. G. (2014). Educational opportunities and learning outcomes of children in Peru: A longitudinal model. En M. Bourdillon y J. Boyden, J. (Eds.), Growing up in poverty: findings from Young Lives (pp. 245-268). Londres: Palgrave Macmillan; Young Lives.

De Belaunde, C. (2011). Profundizando las brechas. Una mirada a la desigualdad en los estudios sobre el sistema educativo peruano. En J. Cotler y R. Cuenca (Ed.), Las desigualdades en el Perú: balances críticos (pp. 273-329). Lima: Instituto de Estudios Peruanos.

Dupriez, V. (2010). Methods of Grouping Learners at School. Paris: Unesco.

Dupriez, V. y Dumay, X. (2006). Inequalities in school systems: Effect of school structure or of society structure? Comparative Education, 42(2), 243-260. https://doi.org/10.1080/03050060600628074

Durlauf, S. (2006). Groups, social influences, and inequality: A memberships theory perspective on poverty traps. En S. Bowles, S. Durlauf y K Hoff (Eds.), Poverty Traps (pp. 141-175). Princeton: Princeton University Press.

Elacqua, G. (2009). The impact of school choice and public policy on segregation: Evidence from Chile. Santiago de Chile: CPCE

(2012). The impact of school choice and public policy on segregation: Evidence from Chile. International Journal of Educational Development, 32(3), 444-453. https://doir.org/10.1016/j.ijedudev.2011.08.003

Ganimian, A. J. (2015). Bajos resultados, altas mejoras. ¿Cómo les fue a los estudiantes peruanos de primaria y secundaria en las últimas evaluaciones internacionales? Lima: Oficina de Medición de la Calidad de los Aprendizajes, Ministerio de Educación del Peru (UMC-Minedu). 
Gasparini, L. C., Jaume, D., Serio, M. y Vázquez, E. (2011). La segregación escolar en Argentina. Reconstruyendo la evidencia. Buenos Aires: CEDLAS.

Gorard, S. (2014). The link between Academies in England, pupil outcomes and local patterns of socio-economic segregation between schools. Research Papers in Education, 29(3), 268-284. https://doi.org/10.1080/02671522.2 014.885726

Gorard, S. y Taylor, C. (2000). A comparison of segregation indices used for assessing the socio-economic composition of schools. Lancaster: Cardiff University - School of Social Sciences. Economic and Social Research Council.

Gorard, S. y Smith, E. (2004). An international comparison of equity ineducation systems. Comparative Education, 40(1), 15-28. https://doi. org/10.1080/0305006042000184863

Guadalupe, C., Burga, A., Miranda, L. y Castillo, L.E. (2015). Brechas de equidad en la Evaluación Censal de Estudiantes 2007-2014: Tres aproximaciones a su medición. Persona, 18, 47-68.

Guadalupe, C., Castillo, L. E., Castro, P., Villanueva, A. y Urquizo, C. (2016). Conclusión de estudios primarios y secundarios en el Peru凶: Progreso, cierre de brechas y poblaciones rezagadas. Lima: Universidad del Pacífico.

Guadalupe, C., León, J., Rodríguez, J.S. y Vargas, S. (2017). Estado de la educación en el Perú. Análisis y perspectivas de la educación básica. Lima: Forge-Grade.

Ireson, J. y Hallam, S. (2001). Ability Grouping in Education. Londres: Paul Chapman Publishing.

Jaume, D. y Gasparini, L.C. (2013). Un estudio sobre el incremento de la segregación escolar en Argentina. Buenos Aires: Cedlas.

Jenkins, S. P., Micklewright, J. y Schnepf, S. V. (2006). Social segregation in secondary schools: How does England compare with other countries? (ISER Working Paper 2006-2). Colchester: University of Essex.

Krüger, N. (2011). The segmentation of the Argentine education system: Evidence from PISA 2009. Regional and Sectoral Economic Studies, 11(3), 41-64.

(2014). Más allá del acceso: Segregación social e inequidad en el sistema educativo argentino. Cuadernos de Economía, 33(63), 513-542. https://doi.org10.15446/cuad.econ.v33n63.45344

(2018). An evaluation of the intensity and impacts of socioeconomic school segregation in Argentina. En Bonal, X. y Bellei, C. (Ed.), Understanding School Segregation. Patterns, Causes and Consequences of Spatial Inequalities in Education (pp. 65-82). Londres: Bloomsbury Academic. 
(2019). La segregación por nivel socioeconómico como dimensión de la exclusión educativa: Quince años de evolución en América Latina. Archivos Analíticos de Politicas Educativas, 27(8), 1-37. http://dx.doi. org/10.14507/epaa.27.3577

León, J. y Collahua, Y. (2016). El efecto del nivel socioeconómico en el rendimiento de los estudiantes peruanos: Un balance de los últimos 15 años. En Grade (Ed.), Investigación para el desarrollo en el Perú: Once balances (pp. 109-162). Lima: Grade.

Lieberson, S. (1981). An asymmetrical approach to segregation. En C. Peach (Ed.), Ethnic segregation in cities (pp. 61-83). Londres: Croom-Helm.

Lisboa Bartholo, T. y da Costa, M. (2018). Patterns of School Segregation in Brazil: Inequalities and Education Policy. En Bonal, X. y Bellei, C. (Ed.), Understanding School Segregation. Patterns, Causes and Consequences of Spatial Inequalities in Education (pp. 179-199). Londres: Bloomsbury Academic.

Ministerio de Educación. (2006). Evaluación Nacional del Rendimiento Estudiantil 2004. ¿Cómo disminuir la inequidad del sistema educativo peruano y mejorar el rendimiento de los estudiantes? Factores explicativos más relevantes en la Evaluación Nacional 2004. Documento de Trabajo 21. Lima: Oficina de Medición de la Calidad de los Aprendizajes, Ministerio de Educación del Peru (UMC-Minedu).

(2016). Marco de fundamentación de las pruebas de la Evaluación Censal de Estudiantes. Reporte Técnico. Lima: Oficina de Medición de la Calidad de los Aprendizajes, Ministerio de Educación del Peru (UMC-Minedu).

(2017). Reporte técnico del estatus socioeconómico en la Evaluación Censal de Estudiantes 2016. Lima: Oficina de Medición de la Calidad de los Aprendizajes, Ministerio de Educación del Peru (UMC-Minedu).

(2018). Evaluación Censal de Estudiantes 2018. Información para directores y docentes. Recuperado de http://umc.minedu. gob.pe/wp-content/uploads/2018/08/Folleto-ECE-2018.pdf

Ministerio de Educación - Oficina de Medición de la Calidad de los Aprendizajes. (2016). Evaluación Censal de Estudios 2016 [base de datos]. Recuperado de http://umc.minedu.gob.pe/resultadosece2016/

Murillo, F. J. (2016). Midiendo la segregación escolar en América Latina. Un análisis metodológico utilizando el Terce. Reice. Revista Iberoamericana sobre Calidad, Eficacia y Cambio en Educación, 14(4), 33-60. https://doi. org/10.15366/reice2016.14.4.002

Murillo, F. J. y Martínez-Garrido, C. (2017a). Estimación de la magnitud de la segregación escolar en América Latina. Magis. Revista Internacional de 
Investigación Educativa, 9(19), 11-30. https://doi.org/10.11144/javeriana. m9-19.emse

(2017b). Segregación escolar por nivel socioeconómico en Ecuador y sus provincias. Runae. Revista Científica de Investigación Educativa de la UAE, 1(2), 31-50.

(2018). Magnitud de la segregación escolar por nivel socioeconómico en España y sus Comunidades Autónomas y comparación con los países de la Unión Europea. RASE. Revista de Sociología de la Educación, 11(1), 37-58. https://doi.org/10.7203/ RASE.11.1.10129

Murillo, F. J., Duk, C. y Martínez-Garrido, C. (2018).Evolución de la segregación socioeconómica de las escuelas de América Latina. Estudios Pedagógicos, 44(1), 157-179. http://dx.doi.org/10.4067/S0718-07052018000100157

Organización para la Cooperación y el Desarrollo Económicos. (2017). Estudio de bienestar y políticas de juventud en el Perú. París: Proyecto OCDE-UE inclusión juvenil

Orfield, G. y Lee, Ch. (2005). Why segregation matters: Poverty and educational inequality. Cambridge, MA.: Harvard University, The Civil Rights Project.

Organización de las Naciones Unidas. (2018). Desafíos y prioridades: Política de adolescentes y jóvenes en el Perú. Lima: Sistema de Naciones Unidas en el Perú.

Rangel, C. (2006). How do schools and neighbourhoods matter for student achievement? A study of educational inequality in Cartagena, Columbia. Springfield: University of Illinois.

Rangel, C. y Lleras, C. (2010). Educational inequality in Colombia: Family background, school quality and student achievement in Cartagena. International Studies in Sociology of Education, 20(4), 291-317. https://doi. org/10.1080/09620214.2010.530855

Reimers, F. (2000). Educación, desigualdad y opciones de política en América Latina en el siglo XXI. Revista Latinoamericana de Estudios Educativos, 30(2), 11-42.

Rojas, M. T. (2009). ¿Es posible imaginar escuelas más integradas socialmente? Docencia, 39, 31-39.

Rivas, A. (2015). América Latina después de PISA: Lecciones aprendidas de la educación en siete países 2000- 2015. Buenos Aires: CIPPEC-Natura.

Sanz, P. (2014). "We don't need the State". A study of the habitus formation process, through school choice, in the Peru's rising middle class. A qualitative study of school choice (Tesis doctoral). Universidad de Bath, UK.

(2015). El tránsito de la escuela pública a la escuela privada en el sector emergente de Lima Metropolitana: ¿Buscando mejor calidad? Revista Peruana de Investigación Educativa, 7, 95-125. 
Unesco. (2008). Los aprendizajes de los estudiantes de América Latina y el Caribe. Primer reporte de los resultados del Segundo Estudio Regional Comparativo y Explicativo. Santiago de Chile: Unesco-Orealc.

(2010). Factores asociados al logro cognitivo de los estudiantes de América Latina y el Caribe. Santiago de Chile: Unesco-Orealc.

(2013). Tercer Estudio Regional Comparativo y Explicativo (Terce). Santiago de Chile: Unesco-Orealc.

Valenzuela, J. P., Bellei, C. y de los Ríos, D. (2008). Evolución de la segregación socioeconómica de los estudiantes chilenos y su relación con el financiamiento compartido. Santiago de Chile: Ministerio de Educación de Chile, Universidad de Chile.

(2010). Segregación escolar en Chile. En S. Martinic y G. Elacqua (Eds.), ¿Fin de ciclo? Cambios en la gobernanza del sistema educativo chileno (pp. 257-284). Santiago de Chile: Unesco-Orealc.

(2014). Socioeconomic school segregation in a market-oriented educational system. The case of Chile. Journal of Education Policy, 29(2), 217-241. https://doi.org/10.1080/0268 0939.2013.806995

Vázquez, E. (2012). Segregación escolar por nivel socioeconómico: Midiendo el fenómeno y explorando sus determinantes. Buenos Aires: Cedlas. 